\title{
Assessment of differential expression of oncogenes in adenocarcinoma of stomach with fluorescent labeling and simultaneous amplification of gene transcripts
}

\author{
Uroš Rajčević ${ }^{1}$, Petra Hudler ${ }^{1}$, Gordan Mijovski ${ }^{2}$, Gregor Gorjanc ${ }^{3}$, \\ Georg Hoelzl ${ }^{4}$, Stanislav Repše ${ }^{5}$, Robert Juvan ${ }^{5}$, Milena Kovač ${ }^{3}$, \\ Christian G. Huber ${ }^{6}$ and Radovan Komel ${ }^{1}$
}

${ }^{1}$ Medical Center for Molecular Biology, Faculty of Medicine, Institute of Biochemistry, Ljubljana, Slovenia; ${ }^{2}$ Clinical Department for Anesthesiology and Intensive Care of Operative Branches, University Clinical Center, Ljubljana, Slovenia; ${ }^{3}$ Department of Animal Science, Biotechnical Faculty, Domzale, Slovenia; ${ }^{4}$ Institute of Analytical Chemistry and Radiochemistry, LeopoldFranzens University, Innsbruck, Austria; ${ }^{5}$ Clinical Department for Abdominal Surgery, University Clinical Center, Ljubljana, Slovenia; ${ }^{6}$ Instrumental Analysis and Bioanalysis, Saarland University, Im Stadtwald, 66123 Saarbruecken, Germany

Background. Gastric cancer is one of the leading malignancies with a poor prognosis and low survival rates. Although the mechanisms underlying its development are still unknown, there is a consensus that genetic instability, inactivation of tumor suppressor genes and over-expression of oncogenes are involved in the early and late stages of gastric carcinogenesis. In the present study we wanted to display differential expression of seven oncogenes, namely CCNE1, EGF, ERBB3, FGF4, HRG1, HGFR and TDGF1.

Patients and methods. We employed a method based on the multiplex reverse transcription polymerase chain (RT-PCR) method with a fluorescence detection.

Results. More than half of patients (74.3\%) out of total 74 with gastric adenocarcinoma had over-expressed at least one oncogene, with the exception of FGF4, which was expressed in tumor tissue of less than one third of patients. $56.8 \%$ of the patients patients showed over-expression of two or more oncogenes.

Conclusions. Patients with precancerous lesions had elevated levels of TDGF1 or cripto-1 (64.9\%) and CCNE1 (57.1\%), suggesting that they could be used as markers for an early detection of malignant changes in stomach. Finally, the fluorescent multiplex RT-PCR method could be of value for rapid assessment of oncogene mRNA levels in small samples of tumor or precancerous biopsies.

Key words: stomach neoplasms - genetics; adenocarcinoma; reverse transcriptase polymerase chain reaction; oncogenes

Received 16 October 2007

Accepted 26 November 2007

Correspondence to: Prof. Radovan Komel, Ph.D. Medical Center for Molecular Biology, Faculty of Medicine, Institute of Biochemistry, Vrazov trg 2, SI1000 Ljubljana, Slovenia. Phone: +386 1543 7644; Fax : +386 154376 41; E-mail: radovan.komel@mf.uni-lj.si

\section{Introduction}

Changes in gene expression are at the core of development and differentiation, and play a decisive role in many pathological processes such as carcinogenesis. Gastric 
cancer (GC) is a leading cause of cancer mortality worldwide, surpassed only by lung and breast cancer. ${ }^{1}$ The leading histological form is adenocarcinoma, accounting for more than $9 / 10$ of all cases, which can be subdivided into two clinicopathological entities: intestinal or well-differentiated and diffuse or poorly differentiated GC. ${ }^{2}$

Recent molecular analyses have clarified many genetic alterations in gastric carcinogenesis but this is hardly sufficient to understand the underlying mechanism(s). ${ }^{3}$ Furthermore, the disease shows diverse clinical properties regarding invasiveness, ability to form metastases and responsiveness to chemotherapy. The transformation of normal gastric epithelial cells into cancer is a multistep process associated with the progressive accumulation of abnormalities in DNA-repair genes, tumor suppressor genes and oncogenes. Increased or excessive expression of oncogenes could, under certain conditions, induce a neoplastic transformation of cells. ${ }^{4}$

A current hypothesis predicts the multistep process of gastric carcinogenesis, which involves, among other, genetic changes of multiple oncogenes. ${ }^{4-6}$ Thus, assessment of expression of putative oncogenes might be of clinical and prognostic importance..$^{7-9}$ Although it was believed that in the sequential multi-step tumorigenesis process, inactivation of a single mutant gene product would be insufficient to obtain tumor regression, recent data proved otherwise. ${ }^{10}$ In mouse models even a transient inactivation of a transforming oncogene (Ras, Myc or Bcr-Abl) was sufficient for reversion of malignant phenotype. ${ }^{10,11}$ Targeting oncogenes could thus be a promising approach in treatment and evidence exists about successful results obtained in humans treated with antibodies for the Her-2 receptor against metastatic breast cancer, and with Bcr-Abl kinase i inhibitor, imatinib mesylate (Glivec), in chronic myelogenous leukemia (CML) and gastrointestinal stromal tumors (GIST). The evidence of oncogene expression could also be useful for selecting the most effective therapy. Recent studies showed that in lung cancer, c-Met (hepatocyte growth factor receptor, HGFR) amplification leads to gefitinib or erlotinib resistance, however, on the other hand, the research on GC cell lines with this defect showed their extreme sensitivity to another type of chemotherapeutics, specific tyrosine kinase inhibitor PHA-665752. ${ }^{7,8}$ Therefore, even though the mechanisms of gastric carcinogenesis are still elusive, it seems that in the future a rapid and reliable assessment of certain key oncogenes might be very important for both diagnosis and treatment. Usually, the study of differential expression of individual oncogenes is a laborious and time consuming procedure. In this work, we developed a diagnostic approach based on fluorescent multiplex quantitative reverse transcription polymerase chain reaction (RT-PCR) assay and capillary electrophoresis separation, which allows insight and relative quantification of differential expression of a number of oncogenes simultaneously. With these two simple techniques seven oncogenes associated with molecular genesis of gastric adenocarcinoma (cyclin E (CCNE1), epidermal growth factor (EGF), fibroblast growth factor 4 (FGF4), teratocarcinoma-derived growth factor 1 or Cripto-1 (TDGF1), hepatocyte growth factor receptor (HGFR) or c-Met, neuregulin 1 or heregulin (HRG1 or NRG1) and v-erb-b2 erythroblastic leukemia viral oncogene homolog 3 (avian) or c-erbB3 (ERBB3)) were screened in samples of tumors and corresponding normal mucosa of 74 patients with adenocarcinoma of the stomach, and in samples of precancerous lesions (chronic atrophic gastritis, ulcers and intestinal metaplasia/dysplasia) and corresponding normal tissues of 77 patients. 


\section{Materials and methods}

\section{Patients}

Samples of tumors and corresponding normal mucosae of 74 patients with GC were contributed by the Clinical Department for Abdominal Surgery, University Clinical Center Ljubljana. Samples of precancerous tissues were obtained at the Clinical Department for Gastroenterology, University Clinical Center Ljubljana. The portions of tumor and precancerous tissues and adjacent non-tumor mucosa were carefully sampled and frozen at $-70^{\circ} \mathrm{C}$ for DNA extraction. Representative portions of tumor samples were also formalin-fixed and paraffin-embedded for immunohistochemistry and histology. A histological evaluation of these samples was confirmed at the Institute of Oncology Ljubljana. Most GC patients were male with 1.6:1 male-to-female ratio. The mean age at the surgery was $64.5 \pm 11.4$ years (mean \pm standard deviation (SD)). The distribution of tumors based on Lauren's classification system in the series included $40(54.8 \%)$ intestinal types, $17(23.3 \%)$ diffuse and $16(21.9 \%)$ cases of mixed type. With respect to Ming's classification, the cases were ranked into 24 (32.9\%) expansive, $35(47.9 \%)$ infiltrative and $13(17.8 \%)$ mixed types. Patients with precancerous lesions were handled as one group, because grouping 11 patients with intestinal metaplasia/dysplasia, 14 cases with ulcers and 52 with chronic atrophic gastritis was not feasible for relevant statistical analysis. The study was approved by the National Medical Ethics Committee and informed consent was obtained from participants. All samples used in the study were anonymized and labeled with an appropriate number.

\section{Total RNA extraction and purification}

Total RNA was extracted from pairs of tissue samples using TRIzol reagent (Invitrogen) or RNeasy Mini Kit (Qiagen) according to manufacturer's instructions. To remove residual impurities such as DNA, proteins and sugars, total RNA was additionally purified using DNAseI (Qiagen), extracted with the mixture of phenol:clorophorm: iso-amyl-alcohol (50:48:2) and precipitated with ethanol. RNA pellet was vacuum-dried and diluted in $8 \mu \mathrm{l}$ of nuclease free, sterile water (Invitrogen). Purity and concentration were assessed spectrophotometricaly (Beckman UD 7500) at $260 \mathrm{~nm}$ and $280 \mathrm{~nm}$. The quality was checked with electrophoresis on agarose gel. For further procedures samples of RNA with the A260/A280 ratio of equal or more than 1.8 and sufficient consistency were applied.

\section{Multiplex RT-PCR}

Primers for the simultaneous amplification of fragments of seven oncogenes (CCNE1, EGF, ERBB3, FGF4, HGFR, HRG1 and TDGF1) were selected from coding sequences using Primer3, web-based primer selection software (Table 1). All sense primers were $5^{\prime}$ end-labeled with one of the fluorescent dyes, 6-FAM ${ }^{\mathrm{TM}}$, TET ${ }^{\mathrm{TM}}$ or HEX $^{\mathrm{TM}}$ (Applied Biosystems). Primers were selected in a manner that the fragments that they amplify did not overlay. ${ }^{8}$ Once the PCR conditions of the 7-gene multiplex reaction were established and optimized, semi-quantitative RT-PCR was performed to study the differential expression of selected oncogenes in both the tumor and normal mucosa specimens. As internal standard (IS) we used a house-keeping gene, gylceraldehyde-3-phosphate dehydrogenase (GAPDH). ${ }^{12}$

For the synthesis of the first chain of complementary DNA (cDNA) 25 pmoles of each of eight non-labeled, antisense primers (CCNE1, EGF, ERBB3, FGF4, HGFR, HRG1, TDGF1) and $1 \mu \mathrm{l}(200 \mathrm{U} / \mu \mathrm{l})$ of Superscript II Reverse transcriptase (Invitrogen) was 
Table 1. Sequences of sense and antisense primers of seven oncogene fragments

\begin{tabular}{|c|c|c|c|}
\hline Oncogene & $\begin{array}{l}\text { Gene name } \\
\text { (Entrez Gene database) }\end{array}$ & Primer sequence $^{a}$ & Product length $\left(\mathbf{b p}^{\mathrm{b}}\right)$ \\
\hline C-met & HGFR & $\begin{aligned} \text { TET }- & \text { 5'-gtgctcctgtttaccttg } \\
& \text { 5'-tagttagtggcggcaccaag }\end{aligned}$ & 291 \\
\hline CR-1 & TDGF1 & $\begin{array}{r}\text { HEX - 5' -gtgtaaatgctggcacggtc } \\
5^{\prime} \text {-aaaggcagatgccagctag }\end{array}$ & 224 \\
\hline Cyclin e & CCNE1 & $\begin{array}{r}\text { HEX - 5'-gtcaagtacaccagccac } \\
\text { 5'-ggatagatatagcagcac }\end{array}$ & 214 \\
\hline C-erbB3 & ERBB3 & $\begin{array}{c}\text { FAM - 5'-cgatgctgagaaccaata } \\
5^{\prime} \text {-acttcccatcgtagacct }\end{array}$ & 171 \\
\hline EGF & EGF & $\begin{array}{r}\text { FAM }-5^{\prime} \text {-gagcgaagctttcatatg } \\
5^{\prime} \text {-tcactgagtcagctccat }\end{array}$ & 159 \\
\hline FGF-4 & FGF4 & $\begin{array}{c}\text { FAM - 5'-caccatgaaggtcacccact } \\
5^{\prime} \text {-cttacacctactcttgca }\end{array}$ & 148 \\
\hline HRG1 & NRG1 & $\begin{aligned} \text { TET }- & 5^{\prime} \text {-tcagcagttcagctccttcc } \\
& 5^{\prime} \text {-ttgtgttgctgtccacttcc }\end{aligned}$ & 141 \\
\hline
\end{tabular}

aPrimer sequences, their fluorescent tags and the lengths of fragments they are amplifying (sense primers are fluorescent tagged); ${ }^{b}$ base pairs.

used. The reverse transcription (RT) reaction was performed following instructions of the producer's protocol (Invitrogen). Next, we performed multiplex PCR, using $1 \mu \mathrm{l}$ of cDNA, $12.34 \mu \mathrm{l}$ of multiplex primer mix, $2.5 \mu 1$ 10x PCR Gold buffer (Applied Biosystems), $1 \mathrm{dNTP}$ mix (2.5 mM each, Promega)), $1 \mu \mathrm{l}$ 4\% DMSO, $0.4 \mu \mathrm{l}$ Taq Gold polymerase (Applied Biosystems) and 3.76 $\mu l$ nuclease free $\mathrm{H}_{2} \mathrm{O}$. PCR conditions were as follows: initial denaturation 10 minutes at $95^{\circ} \mathrm{C}$, followed by 26 reaction cycles; each cycle composed of 30 seconds of denaturation at $95^{\circ} \mathrm{C}, 30$ seconds of annealing at $52^{\circ} \mathrm{C}$ and 3 minutes of elongation at $72^{\circ} \mathrm{C}$. Multiplex reverse transcription and multiplex PCR with fluorescent tagging and simultaneous amplification of gene transcripts were performed separately on GeneAmp PCR system 9600 (Applied Biosystems). The authenticity of fragment amplification with RT-PCR was established by direct sequencing. Following PCR, residual nucleotides, primers, dissociated fluorescent dyes, salts and the enzyme in multiplex RT-PCR reactions were partially purified with QIAquick PCR-product purification kit (Qiagen).
Analysis of RT-PCR products by with capillary electrophoresis with fluorescence detection and by IP-RP-HPLC with UV detection

Purified, fluorescently tagged multiplex RT-PCR products were separated and analyzed with capillary electrophoresis on ABI-PRISM 310 Genetic Analyzer (Applied Biosystems), according to the producer's instructions. GeneScan 500 ${ }^{\circledR}$ Tamra (Applied Biosystems) was used as an internal standard. Results were evaluated with GeneScan software (Applied Biosystems).

Multiplex RT-PCR products were separated, in parallel experiments, also with liquid chromatography. The purpose of this separation was to assess whether the same products can be adequately separated with this alternative technology and whether the results of both techniques would be comparable. Purified non-labeled multiplex RT-PCR products of tumor and normal samples of limited number of patients were separated with ion-pair reversed-phase high performance liquid chromatography (IP-RP-HPLC) on DNASepTM columns (Transgenomic) packed with alkylated nonporous particles 
Table 2. The percentage of patients showing relative expression (RT/RN) of oncogenes in tumors and precancerous lesions

\begin{tabular}{|c|c|c|c|c|}
\hline Oncogenes & $\begin{array}{l}\text { Gene name (Entrez } \\
\text { Gene database) }\end{array}$ & $\begin{array}{l}\mathrm{RT} / \mathrm{RN}>1 \\
\text { (\% of GC } \mathrm{G}^{\mathrm{a}} \text { patients) }\end{array}$ & $\begin{array}{l}\text { RT/RN > } 5 \\
\text { (\% of GC patients) }\end{array}$ & $\begin{array}{l}\mathrm{RT} / \mathrm{RN}>1 \\
\text { (\% of } \mathrm{Pc}^{\mathrm{b}} \text { patients) }\end{array}$ \\
\hline C-met & HGFR & 56.4 & 10.9 & 26.0 \\
\hline CR-1 & TDGF1 & 36.0 & 15.6 & 64.9 \\
\hline Cyclin E & CCNE1 & 42.0 & 15.8 & 57.1 \\
\hline EGF & EGF & 40.0 & 12.7 & - \\
\hline C-erbB-3 & ERBB3 & 44.0 & 16.0 & 13.0 \\
\hline FGF-4 & FGF4 & 59.0 & $-c$ & - \\
\hline HRG1 & NRG1 & 30.3 & 12.1 & 24.7 \\
\hline
\end{tabular}

${ }^{\mathrm{a} G C}$ - gastric cancer; ${ }^{\mathrm{b}} \mathrm{Pc}$ - precancerous lesions; ${ }^{\mathrm{c}}-\mathrm{-}$ - no expression detected.

of polystyrene-divinylbenzene (PS-DVBC18). ${ }^{13}$ The separation solutions were: $\mathrm{A}=$ $0.1 \mathrm{M}$ TEAA + $0.1 \mathrm{mM}$ EDTA and $\mathrm{B}=\mathrm{A}+$ $25 \%$ acetonitrile. Chromatographic conditions for the separation of the multiplex RTPCR products were as follows: $10 \mathrm{~min}$ linear gradient $20-50 \% \mathrm{~B}$ at a flow-rate of 0.75 $\mathrm{ml} / \mathrm{min}$ and $50^{\circ} \mathrm{C}$ column temperature. 15 $\mu \mathrm{l}$ of the sample was injected onto the column in a single injection. Separated amplified fragments of oncogenes were detected with UV-absorbance at $254 \mathrm{~nm}$. Restriction fragments from pBR332 plasmid, digested with HaeIII, were used as size standards.

\section{Assessment of differential expression of oncogenes}

Electropherograms where the height of internal standard/GAPDH was at least 150 relative fluorescence units (RFU) were considered as positive. For relative quantification of differences in expression of seven oncogenes in the samples of tumors and corresponding normal tissues the following method was applied: on the electropherograms representing normal tissue, the peak area of the specific oncogene fragment $(\mathrm{Pn})$ was compared to the peak area of internal standard expressed in normal tissue (ISn). The ratio coming out of this (RN) was a relative value of a specific oncogene transcript abundance in normal tissue. In the same way the relative value of transcript abundance was determined in tumors ( $\mathrm{RT}=\mathrm{Pt} / \mathrm{ISt})$. To determine the relative ratio of specific oncogene expression in tumors and normal tissues, relative values of expression of specific oncogene were compared $(\mathrm{RT} / \mathrm{RN}=(\mathrm{Pt} / \mathrm{ISt}) /(\mathrm{Pn} / \mathrm{ISn}))$.

\section{Results}

Reaction conditions for the multiplex PCR were established gradually by altering individual parameters in the reactions in order to minimize the background on electropherograms (Figure 1). Primers were selected in a manner that the fragments that they amplify did not overlay. In addition, different fluorescent tagging of sense primers served for a better resolution of the amplified fragments. Carefully adjusted melting temperatures of primers and fragments allowed amplification of all the eight fragments at the same annealing temperature and in the same reaction tube.

With a relative quantification of differences in oncogene expression in tumors and corresponding normal tissue at least one oncogene was over-expressed in tumors in most $(74.3 \%)$ of 74 patients, whereas in $25.7 \%$ we did not detect expression of oncogenes under investigation. Thirteen patients $(17.5 \%)$ showed higher levels of one 
oncogene and in forty-two (56.8\%) cases simultaneous over-expression of two or more oncogenes was observed. In $24.3 \%$ of patients we detected 5- or more fold overexpression of individual oncogenes, while $16.2 \%$ showed 5- or more fold over-expression of two or more oncogenes simultaneously. HGFR (c-Met) was over-expressed in $56.4 \%$, TDGF1 in 36\%, CCNE1 in $42 \%$, EGF in $40 \%$, ERBB3 in $44 \%, \mathrm{FGF} 4$ in $59 \%$, and HRG1 (NRG1) in $30.3 \%$ of cases (Table 2).

In tissues of precancerous lesions we established the following expression pattern of investigated oncogenes (Table 2): TDGF1 in $64.9 \%$, cyclin $\mathrm{E}$ in $57.1 \%$, HGFR in $26.0 \%$, HRG1 in $24.7 \%$ and ERBB3 in $13.0 \%$ of examined cases. Interestingly, we did not detect EGF and FGF4 mRNA.

In parallel experiments we separated nonlabeled amplicons of 8 selected patients with HPLC. The results obtained were comparable with the capillary electrophoretic separation of fluorescently labeled products, although we observed that the threshold of UV-absorbance was lower, which is in accordance with previously published results. ${ }^{14}$ Nevertheless, we established that separation of PCR product could be obtained either with HPLC, using non-labeled fragments, thus reducing the cost. However, capillary electrophoresis was more accurate and sensitive, therefore, in our opinion it is a more reliable method, even though there is a need for fluorescently labeled primers, which are slightly more expensive than non-labeled ones.

\section{Discussion}

The assessment of differential expression of multiple oncogenes with multiplex RTPCR and capillary electrophoresis separation was designed as one of approaches for analysis of changes in oncogenes involved in stomach carcinogenesis. We developed and optimized a method for the assess- ment of differential expression of seven oncogenes with fluorescent tagging and simultaneous amplification of oncogene transcripts.

Activated HGFR, encoded by MET protooncogene, was found in $26.0 \%$ of precancerous lesions and in tumors of $56.4 \%$ of GC patients. $10.9 \%$ of the later over-expressed it for 5- or more fold, which is in accordance with the reports of several authors. ${ }^{5,6,15,16}$ It has been shown that deregulation of HGFR is involved in the aberrant turning on of the invasive growth, strongly correlating with a higher metastatic potential of cancer cells and with a poor prognosis in several types of cancer, such as gastric, colorectal, breast, liver, kidney and pancreatic. 9,10,17 Recently, it was confirmed that HGFR activation protects cells from apoptosis, induces motile phenotype in conjunction with beta-catenin and also promotes entry into cell cycle. ${ }^{17}$ Therefore, we could assume that its deregulation is most prominent in the later stages of GC, which was confirmed by our results. Furthermore, MET amplification could constitute an important biomarker for selecting patients for a targeted therapy. Smolen et al. observed that a fraction of GC cell lines appeared to be exquisitely sensitive to a specific MET inhibitor. ${ }^{8}$ TDGF1 or Cripto-1, an epidermal growth factor-CFC (EGF-CFC) family member, was found upregulated in $36 \%$ of our patients with GC and in $65 \%$ precancerous lesions. This gene plays an important role in tumor cell proliferation and migration and is involved in Nodal and presumably also in Wnt, MAPK and PI3K/AKT signaling pathways. ${ }^{18-21}$ Recently, it has been found that Cripto-1 protein is detectable in plasma of breast and colon cancer patients, and even in early pre-malignant lesions of colon, breast and stomach, suggesting its potential use as a biomarker for an early diagnosis. ${ }^{19}$ It is also interesting that Haruma et al. found a correlation of TDGF1 expression with the long- 
term $H$. pylori infection, which is a known risk factor for the GC development. ${ }^{22}$ Interestingly, 8 of 11 patients $(72.7 \%)$ with precancerous lesions with $H$. pylori infection had also up-regulated this gene, which is in agreement with previously reported results. ${ }^{19,22}$ The over-expression of cyclin E in gastric carcinoma was reported by several authors. ${ }^{23-25}$ We found up-regulated cyclin $\mathrm{E}$ in tumors of $42 \%$ of patients; $15.8 \%$ of them over-expressed it 5- or more fold, while in the precancerous tissues it was upregulated in $57.1 \%$ of patients. Moreover, its higher mRNA levels were found in $81.8 \%$ (9 out of 11) patients with $H$. pylori infection. $\mathrm{Yu}$ et al. also found association of cyclin E over-expression with intestinal metaplasia, although it was also associated with invasion, metastasis and prognosis. ${ }^{25,26}$ Our results correlate with these findings. In addition, TDGF1 and cyclin E mRNA levels were among the highest in our precancerous patients, indicating their possible involvement in early changes affecting gastric mucosa, especially in patients with $H$. pylori. Therefore, these two genes are possible candidates for biomarkers for an early diagnosis of malignant changes in stomach epithelium.

EGF and HRG1 are members of a large family of EGF-like growth factors that influence a variety of cellular events, including proliferation, migration and survival. Both these factors bind ERBB3 or ERBB4 through heterodimerization mechanism, producing potent activators of cell transformation. ${ }^{27}$ It has been demonstrated that EGF could activate ERBB3 (and ERBB4) receptors indirectly through EGFR/ERBB3 (or EGFR/ ERBB4) heterodimerization by a transphosphorylation, while on the other hand, HRG proteins act directly as ligands for ERBB family of receptor tyrosine kinases, specifically for ERBB3 and ERBB4. ${ }^{27}$ The expression of ERBB3 and EGF was reported to be elevated in gastric tumors. ${ }^{25,28,29}$ There are few data of HRG1 over-expression in stomach adenocarcinoma, but neoplastic pathologies with HRG1 expression were found in breast, ovary, prostate, small intestine and brain. ${ }^{27}$ The co-expression of these oncogenes might play important roles in malignant potential of GC and precancerous cells. HRGs, ERBB3 and EGF are involved in the regulation of cellular proliferation, differentiation, migration, apoptosis and survival and angiogenesis, therefore, the fine tuning of this cascade most probably directs cell proliferation and differentiation upon the growth factor stimulation, while its deregulation probably plays an important role in the tumor growth. ${ }^{27}$ The elevated levels of all three genes in our GC patients and slightly elevated mRNAs of only ERBB3 and HRG1 in patients with precancerous lesions could also indicate that EGF deregulation appears in the later stages of GC development.

We found slightly higher levels of FGF4 in $59 \%$ of GC patients but in none of the examined cases for 5 - or more times. However, none of the precancerous patients had elevated FGF4, which is in accordance with previous results. ${ }^{25}$ This oncogene might be probably deregulated in the later stages of GC, because it was found that it is mainly implicated in angiogenesis and metastasis. $^{25}$

Multiplex RT-PCR analysis using capillary electrophoresis and laser-induced fluorescence allowed us to quantify a relatively small amounts of mRNAs with high sensitivity. This seems important when dealing with tissue samples obtained from surgery and biopsies. Furthermore, we observed a deregulation of cripto and cyclin E in precancerous patients, thus indicating a possible use of these markers for an early identification of pre-malignant lesions. Researchers have already confirmed the possible use of cripto as a serologic marker for breast and colon carcinoma. ${ }^{18}$ Our method might be 
helpful for a rapid and reliable assessment of expression profiles of selected oncogenes and thus could be used as a tool for selecting appropriate chemotherapy and for the prognosis.

\section{Acknowledgements}

This work was supported by research grant P0-0527-0381 from the Slovenian Ministry for Education, Science and Sports, by ICGEB Cooperative Research Project CRP/ SLO 98-02, and by the Austrian Science Fund (P-14133-PHY), which are gratefully acknowledged.

\section{References}

1. Ferlay J. Globocan 2000: Cancer incidence, mortality, and prevalence worldwide. Lyon: IARC Press; 2001: 1 CD-ROM.

2. Lauren P. The two histological main types of gastric carcinoma: diffuse and so-called intestinal-type carcinoma. An attempt at a histo-clinical classification. Acta Pathol Microbiol Scand 1965; 64: 31-49.

3. Zheng L, Wang L, Ajani J, Xie K. Molecular basis of gastric cancer development and progression. Gastric Cancer 2004; 72: 61-77.

4. Kufe DW, Holland JF, Frei E, Abramson DH, Dang CT, DeAngelis LM, et al. Cancer medicine 6 . Hamilton, Ont.: BC Decker; 2003.

5. Barletta C, Scillato F, Sega FM, Mannella E. Genetic alteration in gastrointestinal cancer. A molecular and cytogenetic study. Anticancer Res 1993; 136A: 2325-9.

6. Tahara E. Genetic pathways of two types of gastric cancer. IARC Sci Publ 2004; 157: 327-49.

7. Engelman JA, Zejnullahu K, Mitsudomi T, Song Y, Hyland C, Park JO, et al. MET amplification leads to gefitinib resistance in lung cancer by activating ERBB3 signaling. Science 2007; 316(5827): 1039-43.
8. Smolen GA, Sordella R, Muir B, Mohapatra G, Barmettler A, Archibald $\mathrm{H}$, et al. Amplification of MET may identify a subset of cancers with extreme sensitivity to the selective tyrosine kinase inhibitor PHA-665752. Proc Natl Acad Sci USA 2006; 103(7): 2316-21

9. Uen YH, Lin SR, Wu CH, Hsieh JS, Lu CY, Yu FJ, et al. Clinical significance of MUC1 and c-Met RT-PCR detection of circulating tumor cells in patients with gastric carcinoma. Clin Chim Acta 2006; 367(1-2): 55-61.

10. Corso S, Comoglio PM, Giordano S. Cancer therapy: can the challenge be MET? Trends Mol Med 2005; 11(6): 84-92.

11. Felsher DW. Cancer revoked: oncogenes as therapeutic targets. Nat Rev Cancer 2003; 3(5): 375-80.

12. Kataoka H, Joh T, Kasugai K, Okayama N, Moriyama A, Asai K, et al. Expression of mRNA for heregulin and its receptor, ErbB-3 and ErbB-4, in human upper gastrointestinal mucosa. Life $\mathrm{Sci}$ 1998; 63(7): 553-64

13. Bachmair F, Huber C, Daxenbichler G. Quanititation of gene expression by means of HPLC analysis of RT-PCR products. Clin Chim Acta 1999; 279(1-2): 25-34.

14. Huber CG. Micropellicular stationary phases for high-performance liquid chromatography of double-stranded DNA. J Chromatogr A 1998; 806: 3-30.

15. Heideman DA, Snijders PJ, Bloemena E, Meijer CJ, Offerhaus GJ, Meuwissen SG, et al. Absence of tpr-met and expression of c-met in human gastric mucosa and carcinoma. J Pathol 2001; 194(4): 42835.

16. Nakajima M, Sawada H, Yamada $Y$, Watanabe A, Tatsumi M, Yamashita J, et al. The prognostic significance of amplification and overexpression of c-met and c-erb B-2 in human gastric carcinomas. Cancer 1999; 85(9): 1894-902.

17. Rasola A, Fassetta M, De Bacco F, D'Alessandro L, Gramaglia D, Di Renzo MF, et al. A positive feedback loop between hepatocyte growth factor receptor and beta-catenin sustains colorectal cancer cell invasive growth. Oncogene 2007; 26(7): 1078-87.

18. Bianco C, Adkins HB, Wechselberger C, Seno M, Normanno N, De Luca A, et al. Cripto-1 activates nodal- and ALK4-dependent and -independent signaling pathways in mammary epithelial Cells. Mol Cell Biol 2002; 22(8): 2586-97. 
19. Bianco C, Strizzi L, Mancino M, Rehman A, Hamada S, Watanabe $\mathrm{K}$, et al. Identification of cripto-1 as a novel serologic marker for breast and colon cancer. Clin Cancer Res 2006; 12(17): 515864.

20. Hamada S, Watanabe K, Hirota M, Bianco C, Strizzi L, Mancino M, et al. beta-Catenin/TCF/ LEF regulate expression of the short form human Cripto-1. Biochem Biophys Res Commun 2007; 355(1): 240-4.

21. Minchiotti G. Nodal-dependant Cripto signaling in ES cells: from stem cells to tumor biology. Oncogene 2005; 24(37): 5668-75.

22. Haruma K, Ito M, Kohmoto K, Kamada T, Kitada Y, Yasui W, et al. Expression of cell cycle regulators and growth factor/receptor systems in gastric carcinoma in young adults: association with Helicobacter pylori infection. Int J Mol Med 2000; 5(2): 185-90.

23. Akama Y, Yasui W, Yokozaki H, Kuniyasu H, Kitahara K, Ishikawa T, et al. Frequent amplification of the cyclin E gene in human gastric carcinomas. Jpn J Cancer Res 1995; 86(7): 617-21.

24. Tenderenda M. A study on the prognostic value of cyclins D1 and E expression levels in resectable gastric cancer and on some correlations between cyclins expression, histoclinical parameters and selected protein products of cell-cycle regulatory genes. J Exp Clin Cancer Res 2005; 24(3): 405-14.

25. Yasui W, Oue N, Aung PP, Matsumura S, Shutoh M, Nakayama H. Molecular-pathological prognostic factors of gastric cancer: a review. Gastric Cancer 2005; 8(2): 86-94.

26. Yu J, Miehlke S, Ebert MP, Szokodi D, Wehvnignh B, Malfertheiner P, et al. Expression of cyclin genes in human gastric cancer and in first degree relatives. Chin Med J (Engl) 2002; 115(5): 710-5.

27. Breuleux M. Role of heregulin in human cancer. Cell Mol Life Sci 2007; 64(18): 2358-77.

28. Kobayashi M, Iwamatsu A, Shinohara-Kanda A, Ihara S, Fukui Y. Activation of ErbB3-PI3-kinase pathway is correlated with malignant phenotypes of adenocarcinomas. Oncogene 2003; 22(9): 1294301.

29. Slesak B, Harlozinska A, Porebska I, Bojarowski T, Lapinska J, Rzeszutko M, et al. Expression of epidermal growth factor receptor family proteins (EGFR, c-erbB-2 and c-erbB-3) in gastric cancer and chronic gastritis. Anticancer Res 1998; 18(4A): 2727-32. 